\title{
Experimental and theoretical investigation of the operating principles of the Figure-9 laser
}

\author{
Ivan Cardea, Svyatoslav Kharitonov, Camille-Sophie Brès \\ Ecole Polytechnique Fédérale de Lausanne (EPFL), Photonic Systems Laboratory, STI-IEL, Station 11, 1015 Lausanne, Switzerland \\ ivan.cardea@epfl.ch
}

\begin{abstract}
We present a theoretical vectorial model, validated by experimental measurements, describing the dependence of the Figure-9 laser output power, operating in continuous wave, on the coupling ratio of the directional coupler. (C) 2018 The Author(s)
\end{abstract}

OCIS codes: $140.3510,140.3430$.

\section{Introduction}

The Figure-9 lasers have recently attracted significant attention. This all-fiber laser architecture is particularly studied for mode-locking operation owing to its ultra-simple configuration incorporating a nonlinear amplifying loop mirror (NALM), which can be used as saturable absorber [1] and can provide pulse pedestal reduction [2]. Recently, dispersive soliton resonance mode-locked Figure-9 laser operating in the thulium band and generating high energy pulses has been demonstrated [3]. Moreover, such laser architecture is potentially low-noise if implemented with polarization maintaining fibres [4]. However, very little efforts have been made to describe the performance and design rules of these lasers, in particular the performance dependence on the coupling ratio of the output coupler. In this paper we present a theoretical vectorial model describing how the output power changes with the coupling ratio of the coupler and with the phase shift acquired by the two counter-propagating waves across the NALM. The model is valiated by experimental measurements.

\section{Theoretical model}

A schematic representation of a Figure-9 laser having a coupler with an intensity cross coupling coefficient $K$ is shown in figure 1a. The cavity consists of a NALM, formed between the input ports of a directional coupler, and a fibre Bragg grating (FBG) connected at one output port of the coupler. The power amplification is provided by the gain unit block (GU), while all the losses within the loop are modelled by the block labelled $l$, which is the power loss coefficient experienced by the two counter-propagating fields.

(a)

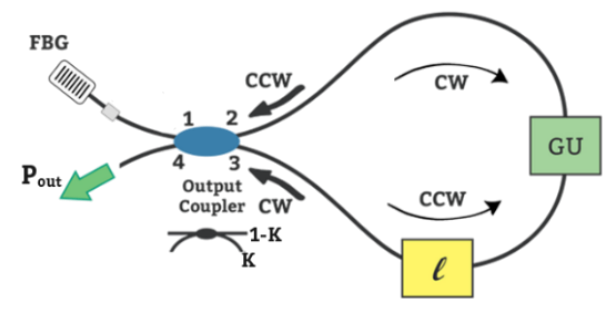

(b)

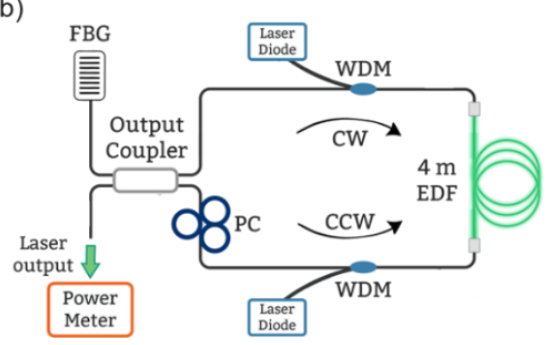

Fig. 1: (a) Layout and (b) experimental setup of the Figure-9 laser. PC, polarization controller; WDM, wavelength division multiplexer; FBG, fibre Bragg grating; EDF, Erbium-doped fibre; CW, clockwise; CCW, counter-clockwise; GU, gain unit.

The theoretical vectorial model is built using the travelling wave approach with the optical field inside the NALM consisting of two counter-propagating waves (clockwise, CW and counter-clockwise, CCW). Their amplitudes depend on the coupling ratio they experience passing through the coupler while their phases are dependent on the birefringence acquired within the loop. Calculating the superposition of the two waves at the coupler and applying the threshold condition for laser oscillation, we obtain the following expression for the output power of the laser:

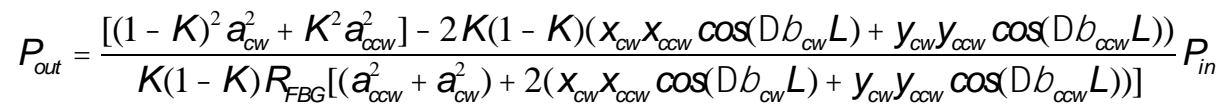

where $x_{c w, c c w}, y_{c w, c c w}$, are the loss coefficients for the $\mathrm{x}$ and y component of the electric fields respectively, $R_{F B G}$ is the reflectivity of the fibre Bragg grating $(\mathrm{FBG}), P_{\text {in }}$ is the intra-cavity power, $L$ is the length of the NALM loop and:

$$
a_{c w}^{2}=x_{c w}^{2}+y_{c w}^{2} \quad a_{c c w}^{2}=x_{c c w}^{2}+y_{c c w}^{2} \quad \quad c w=\begin{array}{ccc}
x & y \\
c w & c w
\end{array}=\begin{array}{ll}
c c w & x \\
c c w
\end{array}
$$


with $\beta_{c w, c c w}^{x}$ and $\beta_{c w, c c w}^{y}$ the propagation constants for the $\mathrm{x}$ and $\mathrm{y}$ component of the electric fields respectively. Finally, in order to apply equation (1) to our laser, the general equation of the amplifier gain was included in the model, obtaining the expression of the intra-cavity power as a function of the pump power and the cavity parameters.

\section{Results and discussion}

The experimental setup of the Figure-9 laser is shown in figure $1 \mathrm{~b}$. The gain unit, composed of $4 \mathrm{~m}$ of erbium doped fibre (EDF) bi-directionally pumped by two $980 \mathrm{~nm}$ laser diodes through two wavelength division multiplexers (WDMs), is placed at the center of the loop. Six directional couplers are used with coupling ratios of 50/50,60/40, $70 / 30,80 / 20,90 / 10$ and $95 / 05$. The use of these six couplers enables us to experimentally investigate coupling coefficient values from 0.05 to 0.95 . A polarization controller (PC) is placed inside the loop in order to maximize or minimize the NALM transmission.

(a)

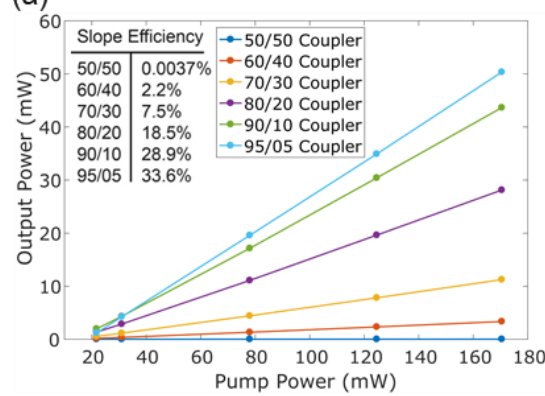

(b)

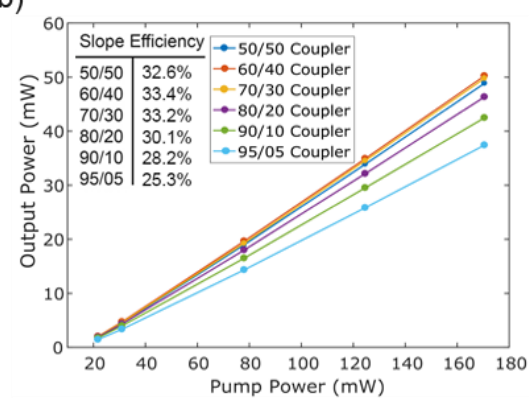

(c)

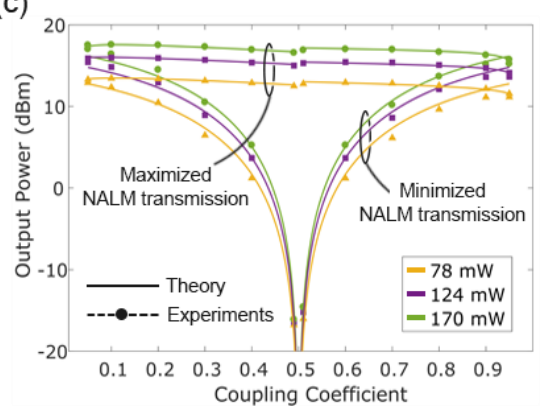

Fig. 2: Experimentally measured output power of the F9 laser. Values as a function of the pump power (a) minimizing and (b) maximizing the NALM transmission; inset table shows the slope efficiency for each configuration, one for each coupler used. (c) Experimental and theoretical curves as a function of the coupling coefficient of the couplers.

All the measurements were performed for different configurations of the laser, one for each coupler. The measurements of the output power collected minimizing and maximizing the NALM transmission for five different pump powers are shown in figure $2 \mathrm{a}$ and $2 \mathrm{~b}$ respectively. It is clear that the slope efficiency (values shown in the inset) increases with increasing coupling coefficient when the NALM transmission is minimized. The configuration with the $95 / 05$ coupler has the best slope efficiency of $33.6 \%$. On the other hand, when the NALM transmission is maximized the slope efficiencies show less variations, ranging from $25.3 \%$ to $33.4 \%$. In this configuration, symmetrical configurations behave with better efficiencies.

The values of the output power are also plotted as a function of the coupling coefficient in figure $2 \mathrm{c}$, for the three highest pump powers, and compared with the curves resulting from our theoretical model. The theoretical curves related to the minimized transmission, were plotted assuming there was not any birefringence in the caviy, i.e. imposing $\Delta \beta_{c w}=\Delta \beta_{c c w}=0$ in equation (1). As shown in fig. $2 \mathrm{c}$, the output power increases with unbalanced coupler and the minimum transmission is reached with the 50/50 coupler. This happens because of the intrinsic effect of the fibre loop mirror, which forces the intra-cavity power to go towards the branch with the FBG.

Experimetnally, in order to maximize the NALM transmission, the PC was adjusted to have a $\pi$ phase difference between the two counter-propagating waves while the theoretical curves were obtained setting $\Delta \beta_{c w}=\Delta \beta_{c c w}=\pi$ in the model and adjusting the loss coefficient for the $\mathrm{x}$ and $\mathrm{y}$ components in order to fit the experimental results. Interestingly, in this case the output power was no longer dependent on the coupling coefficient (fig. 2c) contrary to what was seen minimizing the NALM transmission.

In conclusion, we developed a theoretical vectorial model, validated by experimental measurements, describing the dependence of the Figure-9 laser output power on the coupling ratio of the output coupler and showing that this dependence is different when the NALM transmission is maximized with respect to when it is minimized.

\section{References}

[1] N. J. Smith and N. J. Doran, "Picosecond soliton transmission using concatenated nonlinear optical loop mirror intensity filters," J. Opt. Soc. Am. B 12, 1117-1125 (1995).

[2] I. Gabitov, D. D. Holm and B. P. Luce, "Low-noise picosecond soliton transmission by use of concatenated nonlinear amplifying loop mirrors," J. Opt. Soc. Am. B 14, 1850-1855 (1997).

[3] S. Kharitonov, and C.-S- Brès, “All-Fiber Dissipative Soliton Resonance Mode-locked Figure-9 Thuliumdoped Fiber Laser," in 2017 European Conference on Lasers and Electro-Optics, (Optical Society of America, 2017), paper CJ_13_4.

[4] J. Kim and Y. Song, "Ultralow-noise mode-locked fiber lasers and frequency combs: principles, status, and applications," Adv. Opt. Photon. 8, 465-540 (2016). 